infection by $54-60 \%$. This reduced risk was primarily associated with the $325 \mathrm{mg}$ dose.

Original article Sedlacek M et al. (2007) Aspirin treatment is associated with a significantly decreased risk of Staphylococcus aureus bacteremia in hemodialysis patients with tunneled catheters. Am J Kidney Dis 49: 401-408

\section{Internal snare maneuver restores catheter patency}

Hemodialysis catheter dysfunction often results from formation of a fibrin sheath around the catheter tip and/or from narrowing of the catheter lumen. Fibrin sheaths can be stripped using an external snare, but this procedure requires venous access, local anesthesia and postprocedural observation. Thrombolytic agents can be used to restore lumen patency, but are sometimes ineffective in older thrombi. Other methods for restoring catheter patency also have limitations. Reddy et al. report on the internal snare maneuver, a minimally invasive procedure designed to strip the fibrin sheath from the tip of central venous catheters and to remove thrombi and debris from within catheter lumens.

The internal snare procedure involves the insertion of a loop of $0.089 \mathrm{~cm}$ nitinol guidewire into the catheter. Intraluminal debris and thrombi are removed by moving the snare within the catheter, and the fibrin sheath at the tip is destroyed when the snare emerges and springs open. The procedure is performed on the proximal port followed by the distal port, with continuous cardiac monitoring. No intravenous conscious sedation, and therefore no recovery time, is required.

The procedure was performed on nine catheters in seven patients. No procedure-related complications were reported. One patient was lost to follow-up after 1 week of primary patency. Retrieved clots were between $1 \mathrm{~cm}$ and $5 \mathrm{~cm}$ in length. Initial clinical success (i.e. restoration of target flow rates) was achieved in all seven patients. Primary patency rate was $100 \%$ ( 8 of 8 catheters) at 2, 4 and 6 weeks. At the end of follow-up, primary patency duration ranged from 8 weeks to 40 weeks.

Original article Reddy AS et al. (2007) Fibrin sheath removal from central venous catheters:

an internal snare manoeuvre. Nephrol Dial Transplant 22: $1762-1765$

\section{Does folic acid reduce cardiovascular deterioration in hemodialysis patients?}

Elevated serum total homocysteine (tHcy) level, a risk factor for cardiovascular disease, is prevalent in patients with chronic renal failure. Studies have shown that folic acid reduces tHcy concentrations in people with kidney disease, and Vianna et al. have now investigated whether this folate-induced lowering of tHcy reduces the risk of cardiovascular deterioration.

This double-blind, placebo-controlled study enrolled 186 adults with end-stage renal disease who had been stable on thrice-weekly hemodialysis for at least 4 months. Patients were randomized to receive oral folic acid $10 \mathrm{mg}(n=93)$ or placebo $(n=93)$ thrice-weekly, following dialysis, for 24 months.

At enrollment, median tHcy levels were similarly high in the folate and control groups $(23.5 \mu \mathrm{mol} / \mathrm{l}$ vs $25.8 \mu \mathrm{mol} / \mathrm{l}$, respectively; levels $>13.9 \mu \mathrm{mol} / \mathrm{l}$ are considered abnormal). tHcy concentrations remained high at 6,12 and 24 months in those participants who received placebo, but decreased significantly in folatetreated patients, to a median of $10.5 \mu \mathrm{mol} / \mathrm{l}$ at 24 months $(P<0.01)$.

At 24 months, 15 patients had received kidney transplants and 53 patients had died. There was no statistically significant difference in the incidence of cardiovascular mortality between the folate and placebo groups (17 patients vs 21 patients, respectively), and the incidence of cardiovascular events in the two groups was similar. Mean carotid artery intima-media wall thickness (a predictor of atherosclerotic cardiovascular mortality) decreased in folate-treated patients, and increased in those who received placebo. This finding indicates that early folate supplementation might have some beneficial effects on cardiovascular deterioration in hemodialysis patients.

Original article Vianna ACA et al. (2007) Uremic hyperhomocysteinemia: a randomized trial of folate treatment for the prevention of cardiovascular events. Hemodial Int 11: 210-216

\section{Do diuretics improve the survival of patients on hemodialysis?}

A lack of guidelines on diuretic use in hemodialysis patients has resulted in considerable variation in clinical practice. Bragg-Gresham 